\title{
SIGHTINGS OF DOLPHINS DURING SEISMIC SURVEYS ON THE COAST OF BAHIA STATE, BRAZIL
}

\author{
Lívio Moreira de Gurjão ${ }^{1, *}$, João Eduardo Pereira de Freitas ${ }^{1,2}$ and Douglas Silva Araújo ${ }^{3}$
}

Grant Geophysical Inc. performed 2-D seismic surveys in transition zones on the coast of Bahia State, Brazil, from January to May 2002. The surveys were conducted between Boipeba Island and Barra Grande Beach (Figure 1 ), where depths range from 12 to $55 \mathrm{~m}$. During seismic activities, the occurrence of cetaceans was detected in the surveyed area. The aim of this note is to report sightings of all species of dolphins recorded during seismic surveys conducted in the region.

In order to obtain the appropriate license to operate in Brazilian waters, seismic surveying companies are required by the Brazilian Institute for the Environment and Natural Renewable Resources (IBAMA) to carry out an environmental monitoring program while seismic activities are being conducted. This program was subdivided into two parts, marine and terrestrial monitoring.

Marine monitoring consisted in recording the occurrence of marine organisms from two distinct boats, the gun boat (GB) and the environmental monitoring boat (EMB). The GB was responsible for shooting four 2200-PSI airguns (one shooting cycle/16s) and had a permanent observer on the top of its cabin, who tried to record the presence of those organisms when airguns were either turned on or off ('blind' observation). The EMB had no seismic source onboard; however, an observer was present, with the role of monitoring the operation boundaries to record the presence of marine organisms. Each observer was equipped with a pair of reticule binoculars (red-lens compact Bushmaster $10 \times 25 \mathrm{~mm}$ ) in order to improve his sightings. The GB observer was obligated to stop the seismic surveys whenever either a cetacean or a sea turtle was detected less than $500 \mathrm{~m}$ far from the seismic source of the GB (the safety zone was arbitrarily estimated by the observer).

While the operation was set, for example, as cables were being deployed, the EMB moved away from the surveying site to record the occurrence of marine organisms in the vicinity and/or to place a third observer on land, who was responsible for monitoring the coastline. Once airguns started working, no boat, except the GB, could have its engine on near the surveying site, to avoid interference with the seismic data registering process (performed by a third boat, which recorded the information obtained through the sound-wave reflection on the sea bottom). Hence, the EMB had to keep a distance of at least $1.0 \mathrm{~nm}$ (ca $1.8 \mathrm{~km}$ ) from the shooting line during seismic surveys.

Approximately $6 \mathrm{~km}$ of coastline were surveyed daily, in searching for stranded animals. The position of the airgun shooting line was taken into account to establish the area that would be roamed, and whenever a line was completed, the monitored area changed following the location of the next line to be shot. As strandings were not expected to occur immediately after the GB operation, there was an overlap in the monitored areas in order to optimize monitoring efforts. Thus, one specific site could be monitored during subsequent days after the conclusion of the corresponding shooting line.

Marine monitoring lasted a total of $1225 \mathrm{~h}$, during 112 days (mean $=10.9 \mathrm{~h} /$ day, $\mathrm{SD}=2.8 \mathrm{~h} /$ day, range of 4.0 to $13.8 \mathrm{~h} /$ day - including days without sightings of cetaceans and periods with airguns turned on or off), around three different sites: Boipeba Island, Pratigi Beach and Barra Grande Beach. The daily mean time of airguns turned on was $5.1 \mathrm{~h} /$ day $(\mathrm{SD}=1.9 \mathrm{~h} /$ day, range of 0.2 to $9.0 \mathrm{~h} /$ day), hence, despite the large daily variation, the mean monitoring efforts with airguns turned on and off ('blind' observations) were, respectively, about $5.1 \mathrm{~h} /$ day $(\mathrm{SD}=$ $1.9 \mathrm{~h} /$ day, range of 0.2 to $9.0 \mathrm{~h} /$ day) and $5.8 \mathrm{~h} /$ day $(\mathrm{SD}=$ $1.7 \mathrm{~h} /$ day, range of 3.3 to $8.2 \mathrm{~h} /$ day).

Sightings of two dolphin species were recorded, the marine tucuxi (Sotalia fluviatilis), and the bottlenose dolphin (Tursiops truncatus). Although the field identification of these species can be confusing (Jefferson et al., 1993), they are distinguishable by the longer and narrower snout and the more triangular shape of the dorsal fin of S. fluviatilis, compared to the shorter and more robust snout and the highly falcate dorsal fin of $T$. truncatus (Hetzel and Lodi, 1993). Whenever the identification of the cetacean species was doubtful, dolphins were classified as 'unidentified dolphin' (Table 1). The occurrence of marine tucuxis and bottlenose dolphins in Bahia State, as well as of other 13 cetacean species, had already been reported (Lodi et al., 1990; Hetzel and Lodi, 1993; Reis and Queiroz, 19944; Batista et al., 19985; Sampaio

\footnotetext{
${ }^{1}$ Instituto de Ciências do Mar da Universidade Federal do Ceará (LABOMAR/UFC), Grupo de Ictiologia Marinha Tropical (IMAT). Av. da Abolição, 3207, Meireles, Fortaleza, CE 60165-081 Brazil.

* Corresponding author: Rua Paula Ney, 155/500, Aldeota, Fortaleza, CE 60140-200 Brazil. E-mail: livgurjao@yahoo.com.

${ }^{2}$ E-mail: eduardojpf@hotmail.com.

${ }^{3}$ Grant Geophysical do Brasil Ltda. Rua da Glória, 306, 60 andar, Glória, Rio de Janeiro, RJ 20241-180 Brazil. E-mail: douglas.araujo@grantgeo.com.

${ }^{4}$ Reis, M.S.S. and Queiroz, E.L. (1994) Ocorrência de Stenella cfS. clymene Gray, 1850 (Cetácea, Delphinidae) para o litoral norte do Estado da Bahia, Brasil. Page 142 in Abstracts, VI Reunião de Trabalho de Especialistas em Mamíferos Aquáticos da América do Sul, 24-28 October, Florianópolis, Brazil.

${ }^{5}$ Batista, R.L.G., Santos, U.A. and Reis, M.S.S. (1998) Registros de cetáceos no litoral de Ilhéus-Bahia, Brasil. Page 22 in Abstracts, VIII Reunião de Trabalho de Especialistas em Mamíferos Aquáticos da América do Sul and II Congresso da Sociedade Latinoamericana de Especialistas em Mamíferos Aquáticos, 25-29 October, Olinda, Brazil.
} 
and Reis, 19986; MAMA, 2004). Therefore, the small number of dolphin species identified in the present work does nor reflect the diversity of cetaceans for this region, especially as other cetacean species (e.g., Kogia sp. and Steno bredanensis) have also been recorded during subsequent seismic surveys performed two years later in the same area during longer periods (Araújo, pers. comm.).

Dolphins were sighted in $26 \%$ of the days monitored (46 sightings), and observations of $S$. fluviatilis were much more frequent than those of T. truncatus $(87 \%$ and $0.04 \%$, respectively, see Table 1 ). The fact that marine tucuxis were more often sighted than bottlenose dolphins can simply reflect the inshore distribution of the species. Marine tucuxis occur typically near the coast (Borobia et al., 1991), while bottlenose dolphins can have either coastal or oceanic habits in Brazil (Barreto, 2000). The distribution of these species could make it easier to sight the former than the latter, since seismic activities were conducted in shallow waters.

Group size of marine tucuxis varied from one to 12 animals, and groups of up to four dolphins were recorded in $65 \%$ of the sightings (Table 1). Higher frequencies of small groups of $S$. fluviatilis were also reported for Ceará State, northeastern Brazil (Oliveira et al. 1995), but much larger aggregations of tucuxis have been observed in Rio de Janeiro State, southeastern Brazil (Simão et al. 2000; Lodi, 2003). Calves of S. fluviatilis [identified following Ramos et al. (2000), whose results were later corroborated by Di Beneditto and Ramos (2004)] were present in 4.4\% of the sightings of the species.

Occurrence of marine tucuxis was higher near Boipeba Island (Figure 1). This observation can be biased by the higher sampling effort close to the Boipeba Island (29 groups sighted/600h of survey) in comparison to those next to Pratigi Beach (eight groups sighted/312h) and Barra Grande Beach (three groups sighted/312h). One should also consider that food availability could have influenced dolphins' distribution, since the largest variety of ecosystems, e.g., mangroves, sandy and coral reefs, is found in Boipeba Island. Barros and Wells (1998) stated that distribution and movement of prey influence

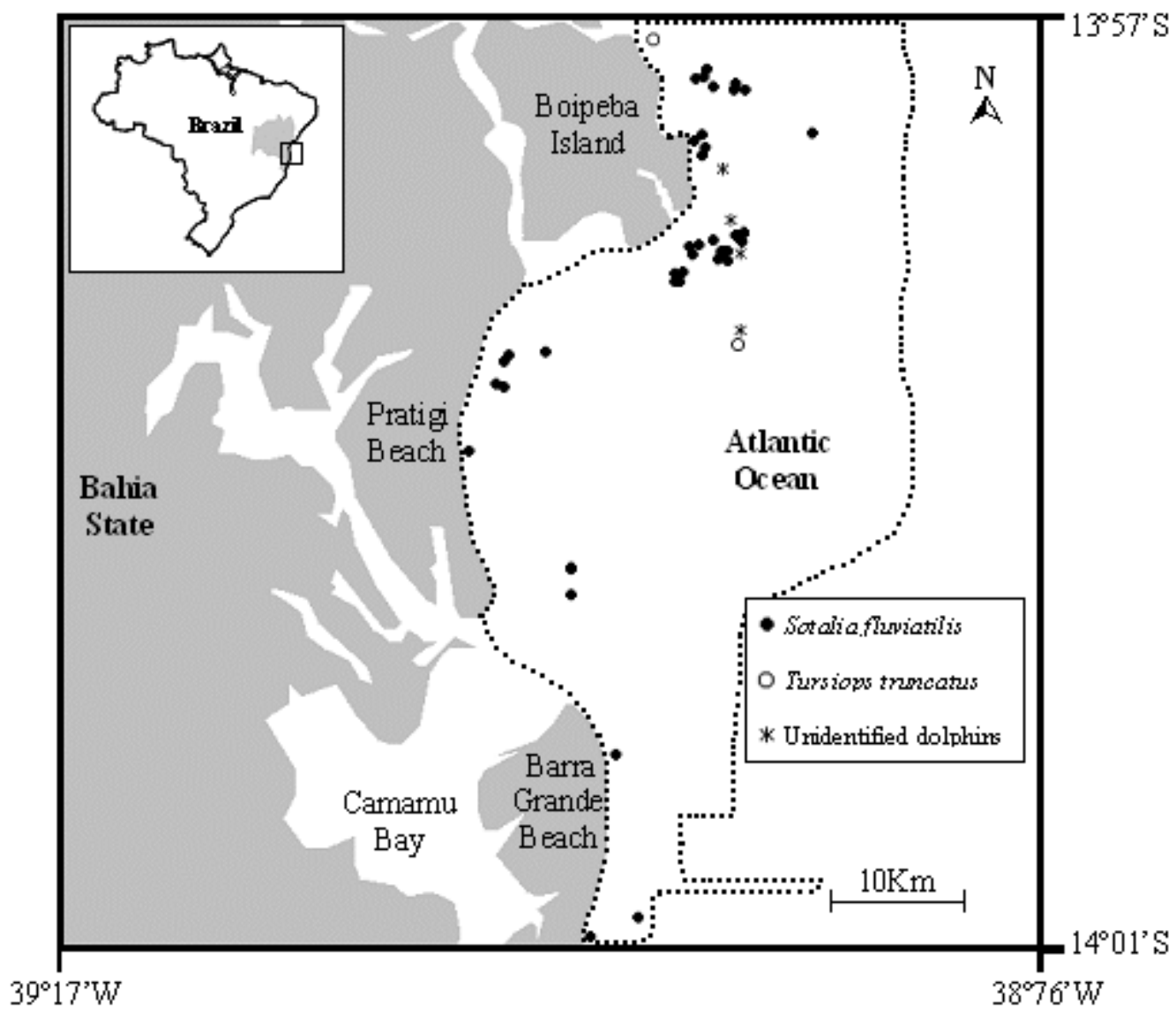

Figure 1. Sightings of dolphins in the area where seismic surveys occurred.

\footnotetext{
${ }^{6}$ Sampaio, C.L.S. and Reis, M.S.S. (1998) Registros de cetáceos na costa nordestina. Page 187 in Abstracts, VIII Reunião de Trabalho de Especialistas em Mamíferos Aquáticos da América do Sul and II Congresso da Sociedade Latinoamericana de Especialistas em Mamíferos Aquáticos, 25-29 October, Olinda, Brazil.
} 
distribution and movement patterns of cetaceans on spatial and temporal scales. Around Pratigi Beach, some S. fluviatilis individuals were sighted near shrimp trawling boats, suggesting some degree of interaction with this kind of fishery.

Bottlenose dolphins were sighted exclusively near Boipeba Island, in two different days (Figure 1). On 25 February 2002, six T. truncatus specimens (four adults and two calves) were observed at $13^{\circ} 34^{\prime} 95^{\prime \prime} \mathrm{S} ; 38^{\circ} 54^{\prime} 50^{\prime \prime} \mathrm{W}$, and, on 7 March 2002, 10 individuals (eight adults and two calves) were sighted at $13^{\circ} 36^{\prime} 22^{\prime \prime} \mathrm{S} ; 38^{\circ} 52^{\prime} 45^{\prime \prime} \mathrm{W}$ (Table 1).

Seismic surveys were never interrupted, since no marine organism was sighted by the GB observer in the safety zone while airguns were turned on. This situation could suggest that, at least apparently, dolphins avoided the noise produced by the airguns; unfortunately, we cannot confirm this statement because no dolphin with suggestive avoiding behavior to airguns was observed. However, it is known that some cetacean species avoid being close to seismic sources. Goold (1996), though using a different methodology, reported that common dolphins, Delphinus delphis, tended to keep a distance from seismic activities when airguns were active.

While seismic activities lasted, neither an injured nor a stranded dolphin was found, and just one dead individual was recorded (on 22 February 2002). The animal could not be identified to species due to the condition of the carcass, which was floating, trapped in a long-line (Table 1). We were not able to conclude whether the dolphin was entangled or not prior to its death, but this kind of observation suggests interaction with fishing activities and no association with the usage of airguns can be made. Although relatively uncommon, incidental catches of small cetaceans in long-lines have been recorded in northeastern Brazil (Siciliano, 1994). Even though some authors reported negative effects of seismic activities on dolphins (Finneran et al., 2002; Gordon et al., 2003), our results do not allow further comments about this topic.

Table 1. List of sightings of dolphins recorded.

\begin{tabular}{|c|c|c|c|c|c|c|}
\hline \multirow{2}{*}{ DATE } & \multirow{2}{*}{ SPECIES } & \multirow{2}{*}{$\mathrm{N}$} & \multicolumn{2}{|c|}{ LOCATION } & \multirow{2}{*}{$\begin{array}{l}\text { LOCAL DEPTH } \\
(\mathrm{m})\end{array}$} & \multirow{2}{*}{ OBSERVER } \\
\hline & & & LATITUDE & LONGITUDE & & \\
\hline 29 January 2002 & Sotalia fluviatilis & 2 & $13^{\circ} 36^{\prime} 57^{\prime \prime S}$ & $38^{\circ} 53^{\prime} 55^{\prime \prime} \mathrm{W}$ & - & $\mathrm{GB}^{* * *}$ \\
\hline 1 February 2002 & Sotalia fluviatilis & 2 & $13^{\circ} 37^{\prime} 38^{\prime \prime} \mathrm{S}$ & $38^{\circ} 53^{\prime} 47^{\prime \prime} \mathrm{W}$ & 5 & EMB \\
\hline 4 February 2002 & Sotalia fluviatilis & 9 & $13^{\circ} 36^{\prime} 16^{\prime \prime S}$ & $38^{\circ} 53^{\prime} 03^{\prime \prime} \mathrm{W}$ & 9 & EMB \\
\hline 4 February 2002 & Sotalia fluviatilis & 2 & $13^{\circ} 41^{\prime} 15^{\prime \prime S}$ & $38^{\circ} 53^{\prime} 34^{\prime \prime} \mathrm{W}$ & 6 & EMB \\
\hline 5 February 2002 & Sotalia fluviatilis & 4 & $13^{\circ} 41^{\prime} 02^{\prime \prime S}$ & $38^{\circ} 53^{\prime} 45^{\prime \prime} \mathrm{W}$ & 5 & EMB \\
\hline 5 February 2002 & Sotalia fluviatilis & 6 & $13^{\circ} 36^{\prime} 13^{\prime \prime S}$ & $38^{\circ} 52^{\prime} 36^{\prime \prime} \mathrm{W}$ & 16 & EMB \\
\hline 6 February 2002 & Sotalia fluviatilis & 4 & $13^{\circ} 38^{\prime} 10^{\prime \prime S}$ & $38^{\circ} 52^{\prime} 40^{\prime \prime} \mathrm{W}$ & 10 & $\mathrm{~GB}^{* * *}$ \\
\hline 6 February 2002 & Sotalia fluviatilis & 12 & $13^{\circ} 34^{\prime} 49^{\prime \prime S}$ & $38^{\circ} 53^{\prime} 59^{\prime \prime} \mathrm{W}$ & - & EMB \\
\hline 7 February 2002 & Sotalia fluviatilis & 10 & $13^{\circ} 41^{\prime} 17^{\prime \prime S}$ & $38^{\circ} 54^{\prime} 04^{\prime \prime} \mathrm{W}$ & 5 & EMB \\
\hline 7 February 2002 & Sotalia fluviatilis & 6 & $13^{\circ} 35^{\prime} 57^{\prime \prime} \mathrm{S}$ & $38^{\circ} 53^{\prime} 16^{\prime \prime} \mathrm{W}$ & - & EMB \\
\hline 10 February 2002 & unidentified dolphin & 2 & $13^{\circ} 43^{\prime} 09^{\prime \prime S}$ & $38^{\circ} 53^{\prime} 23^{\prime \prime} \mathrm{W}$ & - & EMB \\
\hline 14 February 2002 & unidentified dolphin & 1 & $13^{\circ} 38^{\prime} 29^{\prime \prime S}$ & $38^{\circ} 52^{\prime} 41^{\prime \prime} \mathrm{W}$ & - & EMB \\
\hline 14 February 2002 & Sotalia fluviatilis & 2 & $13^{\circ} 36^{\prime} 11^{\prime \prime S}$ & $38^{\circ} 52^{\prime} 60^{\prime \prime} \mathrm{W}$ & - & EMB \\
\hline 14 February 2002 & Sotalia fluviatilis & 1 & $13^{\circ} 36^{\prime} 11^{\prime \prime S}$ & $38^{\circ} 52^{\prime} 60^{\prime \prime} \mathrm{W}$ & - & EMB \\
\hline 14 February 2002 & Sotalia fluviatilis & 7 & $13^{\circ} 36^{\prime} 11^{\prime \prime S}$ & $38^{\circ} 52^{\prime} 60^{\prime \prime} \mathrm{W}$ & - & EMB \\
\hline 15 February 2002 & Sotalia fluviatilis & 3 & $13^{\circ} 40^{\prime} 51^{\prime \prime S}$ & $38^{\circ} 55^{\prime} 84^{\prime \prime} \mathrm{W}$ & 4 & $\mathrm{~GB}^{* * *}$ \\
\hline 15 February 2002 & Sotalia fluviatilis & 3 & $13^{\circ} 38^{\prime} 96^{\prime \prime} \mathrm{S}$ & $38^{\circ} 51^{\prime} 83^{\prime \prime} \mathrm{W}$ & - & EMB \\
\hline 16 February 2002 & unidentified dolphin & 1 & $13^{\circ} 40^{\prime} 05^{\prime \prime S}$ & $38^{\circ} 53^{\prime} 21^{\prime \prime} \mathrm{W}$ & 7 & EMB \\
\hline 17 February 2002 & Sotalia fluviatilis & 1 & $13^{\circ} 43^{\prime} 62^{\prime \prime} \mathrm{S}$ & $38^{\circ} 57^{\prime} 73^{\prime \prime} \mathrm{W}$ & - & EMB \\
\hline 19 February 2002 & Sotalia fluviatilis & 2 & $13^{\circ} 41^{\prime} 68^{\prime \prime} \mathrm{S}$ & $38^{\circ} 54^{\prime} 18^{\prime \prime} \mathrm{W}$ & - & EMB \\
\hline 20 February 2002 & Sotalia fluviatilis & 2 & $13^{\circ} 40^{\prime} 59^{\prime \prime} \mathrm{S}$ & $38^{\circ} 53^{\prime} 17^{\prime \prime} \mathrm{W}$ & 15 & $\mathrm{~GB}^{* * *}$ \\
\hline 21 February 2002 & Sotalia fluviatilis & 2 & $13^{\circ} 41^{\prime} 12^{\prime \prime S}$ & $38^{\circ} 53^{\prime} 57^{\prime \prime} \mathrm{W}$ & - & EMB \\
\hline 21 February 2002 & Sotalia fluviatilis & 7 & $13^{\circ} 40^{\prime} 45^{\prime \prime} \mathrm{S}$ & $38^{\circ} 53^{\prime} 12^{\prime \prime} \mathrm{W}$ & - & EMB \\
\hline 22 February 2002 & Sotalia fluviatilis & 1 & $13^{\circ} 32^{\prime} 02^{\prime \prime} \mathrm{S}$ & $38^{\circ} 48^{\prime} 74^{\prime \prime} \mathrm{W}$ & - & $\mathrm{GB}^{* * *}$ \\
\hline 22 February 2002 & unidentified dolphin & $1^{* *}$ & $13^{\circ} 33^{\prime} 41^{\prime \prime S}$ & $38^{\circ} 48^{\prime} 23^{\prime \prime} \mathrm{W}$ & 40 & EMB \\
\hline 24 February 2002 & Sotalia fluviatilis & 3 & $13^{\circ} 37^{\prime} 39^{\prime \prime S}$ & $38^{\circ} 48^{\prime} 89^{\prime \prime} \mathrm{W}$ & - & EMB \\
\hline
\end{tabular}


conclusion

\begin{tabular}{|c|c|c|c|c|c|c|}
\hline \multirow{2}{*}{ DATE } & \multirow{2}{*}{ SPECIES } & \multirow{2}{*}{$\mathrm{N}$} & \multicolumn{2}{|c|}{ LOCATION } & \multirow{2}{*}{$\begin{array}{l}\text { LOCAL DEPTH } \\
(\mathrm{m})\end{array}$} & \multirow{2}{*}{ OBSERVER } \\
\hline & & & LATITUDE & LONGITUDE & & \\
\hline 25 February & Tursiops truncatus & $6^{*}$ & & & & EMB \\
\hline 2002 & & & $13^{\circ} 34^{\prime} 95^{\prime \prime} \mathrm{S}$ & $38^{\circ} 54^{\prime} 50^{\prime \prime} \mathrm{W}$ & 10 & \\
\hline 25 February & Sotalia fluviatilis & & & & & EMB \\
\hline 2002 & & 8 & $13^{\circ} 35^{\prime} 90^{\prime \prime} \mathrm{S}$ & $38^{\circ} 53^{\prime} 43^{\prime \prime} \mathrm{W}$ & 13 & \\
\hline 1 March 2002 & Sotalia fluviatilis & $8^{*}$ & $13^{\circ} 45^{\prime} 93^{\prime \prime} \mathrm{S}$ & $38^{\circ} 59^{\prime} 68^{\prime \prime} \mathrm{W}$ & - & EMB \\
\hline 1 March 2002 & Sotalia fluviatilis & 6 & $13^{\circ} 50^{\prime} 23^{\prime \prime} \mathrm{S}$ & $38^{\circ} 56^{\prime} 95^{\prime \prime} \mathrm{W}$ & 7 & EMB \\
\hline 1 March 2002 & Sotalia fluviatilis & 4 & $13^{\circ} 49^{\prime} 26^{\prime \prime} \mathrm{S}$ & $38^{\circ} 56^{\prime} 80^{\prime \prime} \mathrm{W}$ & - & EMB \\
\hline 2 March 2002 & Sotalia fluviatilis & $2^{*}$ & $13^{\circ} 44^{\prime} 53^{\prime \prime} S$ & $38^{\circ} 58^{\prime} 75^{\prime \prime} \mathrm{W}$ & - & EMB \\
\hline 7 March 2002 & Tursiops truncatus & $10^{*}$ & $13^{\circ} 36^{\prime} 22^{\prime \prime} \mathrm{S}$ & $38^{\circ} 52^{\prime} 45^{\prime \prime} \mathrm{W}$ & - & EMB \\
\hline 10 March 2002 & Sotalia fluviatilis & 3 & $13^{\circ} 40^{\prime} 01^{\prime \prime} \mathrm{S}$ & $38^{\circ} 56^{\prime} 32^{\prime \prime} \mathrm{W}$ & - & EMB \\
\hline 11 March 2002 & Sotalia fluviatilis & 4 & $13^{\circ} 37^{\prime} 48^{\prime \prime} \mathrm{S}$ & $38^{\circ} 53^{\prime} 01^{\prime \prime} \mathrm{W}$ & - & EMB \\
\hline 15 March 2002 & Sotalia fluviatilis & 3 & $13^{\circ} 40^{\prime} 27^{\prime \prime} \mathrm{S}$ & $38^{\circ} 56^{\prime} 25^{\prime \prime} \mathrm{W}$ & - & EMB \\
\hline 17 March 2002 & Sotalia fluviatilis & 7 & $13^{\circ} 43^{\prime} 52^{\prime \prime S}$ & $38^{\circ} 57^{\prime} 30^{\prime \prime} \mathrm{W}$ & - & EMB \\
\hline 21 March 2002 & Sotalia fluviatilis & 2 & $13^{\circ} 43^{\prime} 60^{\prime \prime} \mathrm{S}$ & $38^{\circ} 58^{\prime} 62^{\prime \prime} \mathrm{W}$ & 6 & EMB \\
\hline 23 March 2002 & Sotalia fluviatilis & 3 & $13^{\circ} 41^{\prime} 66^{\prime \prime} \mathrm{S}$ & $38^{\circ} 54^{\prime} 19^{\prime \prime} \mathrm{W}$ & 7 & EMB \\
\hline 24 March 2002 & Sotalia fluviatilis & 4 & $13^{\circ} 41^{\prime} 39^{\prime \prime} \mathrm{S}$ & $38^{\circ} 55^{\prime} 06^{\prime \prime} \mathrm{W}$ & - & EMB \\
\hline 25 March 2002 & Sotalia fluviatilis & 2 & $13^{\circ} 40^{\prime} 17^{\prime \prime} \mathrm{S}$ & $38^{\circ} 56^{\prime} 56^{\prime \prime} \mathrm{W}$ & 4 & EMB \\
\hline 31 March 2002 & Sotalia fluviatilis & 3 & $13^{\circ} 41^{\prime} 23^{\prime \prime} \mathrm{S}$ & $38^{\circ} 57^{\prime} 30^{\prime \prime} \mathrm{W}$ & - & EMB \\
\hline 13 April 2002 & Sotalia fluviatilis & 12 & $13^{\circ} 59^{\prime} 56^{\prime \prime} \mathrm{S}$ & $38^{\circ} 56^{\prime} 43^{\prime \prime} \mathrm{W}$ & - & EMB \\
\hline 28 April 2002 & Sotalia fluviatilis & 10 & $13^{\circ} 54^{\prime} 42^{\prime \prime} \mathrm{S}$ & $38^{\circ} 55^{\prime} 36^{\prime \prime} \mathrm{W}$ & 12 & EMB \\
\hline 4 May 2002 & Sotalia fluviatilis & 8 & $13^{\circ} 37^{\prime} 22^{\prime \prime S}$ & $38^{\circ} 50^{\prime} 54^{\prime \prime} \mathrm{W}$ & 25 & $\mathrm{~GB}^{* * *}$ \\
\hline 8 May 2002 & Sotalia fluviatilis & 3 & $13^{\circ} 41^{\prime} 39^{\prime \prime} \mathrm{S}$ & $38^{\circ} 54^{\prime} 54^{\prime \prime} \mathrm{W}$ & - & $\mathrm{GB}^{* * *}$ \\
\hline
\end{tabular}

Depths were determined by sonar. The GB had its own equipment and estimated depths on the EMB were obtained by requesting this information from the nearest boat equipped with a sonar. $\left(^{*}\right)$ presence of calves, $\left({ }^{* *}\right)$ dead dolphin - entangled in a long-line, $(-)$ no data available, (GB) Gun Boat, (EMB) Environmental Monitoring Boat, $\left({ }^{* * *}\right)$ airguns turned off.

\section{Acknowledgements}

The authors would like to thank Grant Geophysical Inc. for logistical support and permission to publish the data presented here. We are also thankful to Dr. Renata Ramos, Cristiano Parente, Dr. Nélio B. Barros and an anonymous referee for reviewing this note and providing important criticism to improve it.

\section{References}

Barreto, A.S. (2000) Variação Craniana e Genética de Tursiops truncatus (Delphinidae, Cetacea) na Costa Atlântica da América do Sul. Doctoral Thesis, Fundação Universidade do Rio Grande: Rio Grande. 111pp.

Barros, N.B. and Wells, R.S. (1998) Prey and feeding patterns of resident bottlenose dolphins (Tursiops truncatus) in Sarasota Bay, Florida. Journal of Mammalogy 79(3): 1045-1059.

Borobia, M., Siciliano, S., Lodi, L. and Hoek, W. (1991) Distribution of the South American dolphin Sotalia fluviatilis. Canadian Journal of Zoology 69: 1025-1039.

Di Beneditto, A.P. and Ramos, R. (2004) Biology of the marine tucuxi dolphin (Sotalia fluviatilis) in south-eastern Brazil.
Journal of the Marine Biological Association of the United Kingdom 84: 1245-1250.

Finneran, J.J., Schlundt, C.E., Dear, R., Carder, D.A. and Ridgway, S.H. (2002) Temporary shift in masked hearing thresholds in odontocetes after exposure to single underwater impulses from a seismic watergun. The Journal of the Acoustical Society of America 111(6): 2929-2940.

Goold, J.C. (1996) Acoustic assessment of populations of common dolphin Delphinus delphis in conjunction with seismic surveying. Journal of the Marine Biological Association of the United Kingdom 76(3): 811-820.

Gordon, J., Gillespie, D., Potter, J., Frantzis, A., Simmonds, M.P., Swift, R. and Thompson, D. (2003) A review of the effects of seismic surveys on marine mammals. The Marine Technology Society Journal 37(4): 16-34.

Hetzel, B. and Lodi, L. (1993) Baleias, botos e golfinhos: guia de identificação para o Brasil. Editora Nova Fronteira, Rio de Janeiro, Brazil.

Jefferson, T.A., Leatherwood, S. and Webber, M.A. (1993) Marine mammals of the world. Ed. FAO, Rome.

Lodi, L. (2003) Tamanho e composição de grupo dos botoscinza, Sotalia guianensis (van Bénéden, 1864) (Cetacea, Delphinidae), na Baía de Paraty, Rio de Janeiro, Brasil. Atlântica 25(2): 135-146. 
Lodi, L.F., Siciliano, S. and Capistrano, L. (1990) Mass stranding of Peponocephala electra (Cetacea, Globicephalinae) on Piracanga beach, Bahia, northeastern Brazil. Scientific Reports of Cetacean Research 1: 79-84.

MAMA (2004) Projeto MAMA: mamíferos marinhos. http:// www.lbm.com.br/mama.

Oliveira, J.A., Ávila, F.J.C., Alves Júnior, T.T., Furtado Neto, M.A.A. and Monteiro Neto, C. (1995) Monitoramento do boto cinza, Sotalia fluviatilis (Cetacea: Delphinidae) em Fortaleza, Estado do Ceará, Brasil. Arquivo de Ciências do Mar 29(1-2): 28-35.
Ramos, R., Di Beneditto, A.P. and Lima, N.R.W. (2000) Growth parameters of Pontoporia blainvillei and Sotalia flutivatilis (Cetacea) in northern Rio de Janeiro, Brazil. Aquatic Mammals 26: 65-75.

Siciliano, S. (1994) Review of small cetaceans and fishery interactions in coastal waters of Brazil. Report of the International Whaling Commission 15: 241-250.

Simão, S.M., Pizzorno, J.L.A, Perry, V.N. and Siciliano S. (2000) Aplicação da técnica de fotoidentificação do botocinza, Sotalia fluviatilis fluviatilis (Cetacea, Delphinidae) da Baía de Sepetiba. Floresta e Ambiente 7(1): 31-39. 